\title{
The Study of the Asymmetric Multiple Encounters Problem and Its Application to Obtain Jupiter Gravity Assisted Maneuvers
}

\author{
Denilson Paulo Souza dos Santos, Antônio Fernando Bertachini de Almeida Prado, \\ and Evandro Marconi Rocco
}

Division of Space Mechanics and Control, INPE, C.P. 515, 12227-310 São José dos Campos, SP, Brazil

Correspondence should be addressed to Antônio Fernando Bertachini de Almeida Prado; prado@dem.inpe.br

Received 7 November 2012; Revised 31 March 2013; Accepted 3 April 2013

Academic Editor: Silvia Maria Giuliatti Winter

Copyright (C) 2013 Denilson Paulo Souza dos Santos et al. This is an open access article distributed under the Creative Commons Attribution License, which permits unrestricted use, distribution, and reproduction in any medium, provided the original work is properly cited.

\begin{abstract}
The Multiple Encounters Problem is described in the literature as the problem of finding trajectories for a spacecraft that leaves from a mother planet, describes a trajectory in the interplanetary space, and then goes back to the mother planet. The present paper extends the literature and the departure and arrival angles of the spacecraft are generalized to be nonsymmetrical. The solutions are shown in terms of the true $(\nu)$ and eccentric anomaly $(\eta)$. The velocity variation $(\Delta V)$ required for the transfer is also shown. Then, this study is generalized to consider the possibility that the spacecraft makes a close approach with the mother planet to change its energy in the return trip. The velocity $(\Delta V)$ and energy variation $(\Delta E)$ due to this passage are obtained. The topics studied here can be applied in missions that leave and come back to the Earth, with the goal of studying the interplanetary space, as well as for missions whose objective is to make an alteration in the energy of the space vehicle through a swing-by with the mother body.
\end{abstract}

\section{Introduction}

The literature is extensive with respect to problems involving transfer orbits and optimal spacecraft maneuvers. Goddard [1], in 1919, was a pioneer in studying the problem of transferring a spacecraft between two points. In his research, approximated solutions are proposed for the problem of sending a rocket to high altitudes minimizing the fuel consumed. Regarding orbital transfers, a classic work was made by Hohmann [2], in 1925. The problem of transferring a spacecraft between two coplanar circular orbits with free time around a central force field (Newtonian) was studied, obtaining as a solution a bi-impulsive elliptical transfer orbit. This solution is used even today, for a first mission analysis, and was considered as the final solution of the problem up to the year 1959, when Shternfeld [3] and Hoelker and Silber [4] showed that a three-impulsive transfer is more economical, depending on the final and initial orbits involved. Several other papers studying impulsive maneuvers to change the orbit of a spacecraft can be found in [5-19]. Later, the idea of a nonimpulsive thrust was considered to solve this orbital maneuver problem. Lawden $[20,21]$ described a theoretical complete solution for the problem of orbital transfer between two points with minimum fuel consumption. This problem received the name "Lawden's Problem" due to its commitment to solve it. He introduced a new concept, "the primer vector," which is the Lagrange multiplier associated with the velocity vector, to find a necessary condition for optimal impulsive trajectories according to the magnitude of this vector. After that, many results appeared in the literature related to this type of maneuvers, as shown in [22-34].

Regarding the Multiple Encounters Problem, it was introduced in the literature by Hénon [35], in 1968, that studied a maneuver where the spacecraft starts its motion in a mother body and then returns to this same body, after a journey in a different trajectory with respect to this mother body. He demonstrated that the solution can be reduced to simple algebraic equations. This maneuver became known as "Hénon's Transfer" (Prado and Broucke [36, 37]). Orbits of this type were also examined by Bruno [38], Perko [39], and Hitzl and Hénon [40, 41] considering the circular restricted three-body problem as the mathematical model. In particular, $[40,41]$ investigated the importance of the role of the Jacobian constant in families of orbits for the case $\mu=0$ 
and the stability in the vicinity of the orbits with $\mu=0$, where $\mu$ is the mass of the secondary body in canonical units. They sought to find consecutive meetings between $M_{3}$ and the orbit of $M_{2}$ in the restricted three-body problem for the case $\mu=0$. Later, Howell [42] generalized this study to include elliptical orbits for the primaries, and Santos [43] and Santos et al. [44] considered angles with different magnitudes for the initial and final points of the transfer.

The problem studied here is the transfer of a spacecraft from one body back to the same body, with a possible swing-by in the return passage of the spacecraft. There are numerous applications of this problem, such as (i) to transfer a spacecraft from the Earth to an interplanetary trip and then back to the Earth, without the need of maneuvers during this process, implying the optimization of the fuel consumption; (ii) to transfer a spacecraft from the Moon, which may include a passage by the Earth, and then put it back on the Moon; (iii) rendezvous maneuvers, when one desires that the space vehicle stands alongside another spacecraft; (iv) to maneuver the spacecraft so that it leaves and return to the Earth to perform a swing-by to change its energy, and then go to the outer Solar System; (v) to move a spacecraft that is around a planet, like Jupiter, to another location in the Solar System or even out to the interstellar space.

For these last two applications, it is necessary to combine the Multiple Encounters Problem with the gravity assisted maneuvers. This type of maneuver is used very often in interplanetary trajectories. It uses a close approach with a celestial body to change the orbit of a spacecraft. References [45-61] show some applications of that problem. Details of the combination of those two problems are better explained later in this paper.

\section{Description of the Multiple Encounters Problem}

To describe the Multiple Encounters Problem, let us call $M_{1}$ and $M_{2}$ the two primary bodies of the system, with masses $(1-\mu)$ and $\mu$, respectively. $M_{2}$ is in a circular orbit (in the original version of the problem studied by Hénon [35]) or in an elliptical orbit (in the extension made by Howell [42]) around $M_{1}$. Hénon [35] studied this problem and published solutions for the case of circular orbits. Howel [42] published solutions for the elliptic case, where the transfers were symmetrical with respect to the periapsis. Prado and Broucke [36, 37] also published solutions for this problem, in the same situations, using the Lambert method to solve the problem.

The spacecraft $M_{3}$ (the third body) leaves $M_{2}$ at the point $P\left(t=\psi_{0}\right)$, follows a trajectory around $M_{1}$, and then meets again with $M_{2}$, at the point $Q\left(t=\psi_{f}\right)$, where $\psi_{0}, \psi_{f} \in[0,2 \pi]$ with $\psi_{f}>\psi_{0}$ (Figure 1). The literature usually studied this problem under the condition $\psi_{f}=-\psi_{0}$, but now this study is extended to consider the cases where $\psi_{0}$ and $\psi_{f}$ are not necessarily symmetrical [43]. A priori, it is assumed that $\mu_{2}=$ 0 (disregard the effects of the attraction of $M_{2}$ on $M_{3}$ ), which reduces the problem of three bodies to the two-body problem. So, it is possible to use Kepler's equations in the development

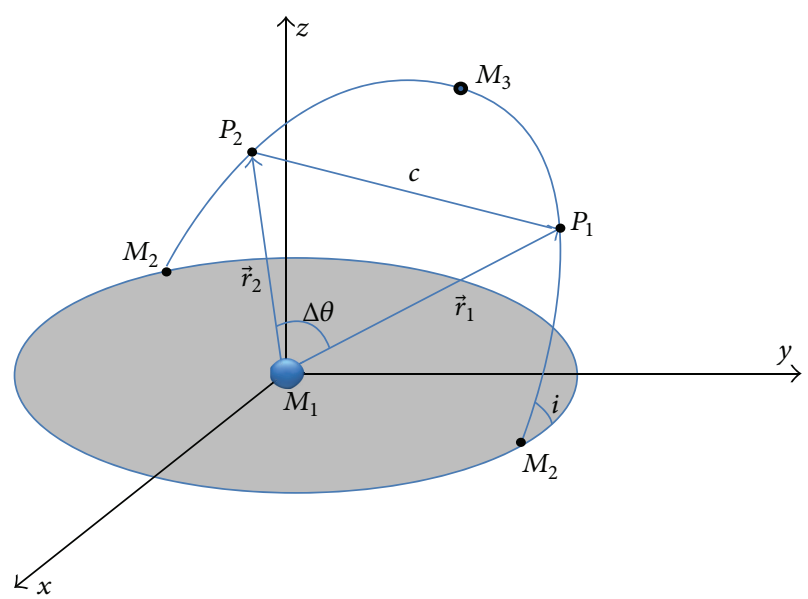

Figure 1: The geometry of Hénon's problem formulated as Lambert problem [43].

of the solutions. Two impulses will be used to perform the transfer maneuver; the first one makes the spacecraft to leave the mother body, and the second one is used to capture the spacecraft again. Figure 1 illustrates this situation, where the $x-y-z$ system of reference is any inertial system. The $M_{3}$ body does not escape from the gravitational attraction of $M_{1}$ and has negligible mass.

Lambert's problem can be formulated as follows. "Find an unperturbed orbit under the mathematical model given by a law that works with the inverse square of the distance (Newtonian formulation), that connects two given points $P$ and $Q$, with the transfer time $(\Delta t)$ specified." In the literature, several researchers have solved this problem using distinct formulations. From a geometrical analysis of the problem $[36,37]$, it is possible to write the equations shown next.

(i) The position coordinates of $M_{2}$ are defined as (assuming circular orbits, in the geocentric system)

$$
\begin{aligned}
& x_{2}=\cos (t), \\
& y_{2}=\sin (t) .
\end{aligned}
$$

(ii) The position coordinates of $M_{3}$ are defined as (assuming coplanar elliptic orbits)

$$
\begin{aligned}
& x_{3}=a(\cos (E)-e), \\
& y_{3}=a \sqrt{1-e^{2}} \sin (E), \\
& t=a^{3 / 2}(E-e \sin (E)),
\end{aligned}
$$

where $(a, e$, and $E$ ) are the Keplerian elements semimajor axis, eccentricity and eccentric anomaly of the orbit of the body, and $t$ is the time. 
There are different possibilities for the orbit of $M_{3}$ around $M_{1}$ [44]. Analyzing them, it is possible to define [36, 37]

$$
\begin{gathered}
\varepsilon=\left\{\begin{array} { l } 
{ + 1 , } \\
{ - 1 , }
\end{array} \text { if the periapsis is in an abscissa } \left\{\begin{array}{l}
\text { positive } \\
\text { negative }
\end{array}\right.\right. \\
\mathcal{\varepsilon}^{\prime}=\left\{\begin{array} { l } 
{ + 1 , } \\
{ - 1 , }
\end{array} \text { if the orbit sense is } \left\{\begin{array}{l}
\text { progressive }, \\
\text { retrograde },
\end{array}\right.\right.
\end{gathered}
$$

$\varepsilon^{\prime \prime}=\left\{\begin{array}{l}+1, \\ -1,\end{array}\right.$ if the passage by $\psi=0$ is at the $\left\{\begin{array}{l}\text { periapsis, } \\ \text { apoapsis. }\end{array}\right.$

Using this formulation, the generalized position coordinates of $M_{3}$ are

$$
\begin{gathered}
x_{3}=\varepsilon a\left(\varepsilon^{\prime} \cos (E)-e\right), \\
y_{3}=\varepsilon^{\prime} a \sqrt{1-e^{2}} \varepsilon^{\prime \prime} \sin (E), \\
t=a^{3 / 2}\left(E-\varepsilon^{\prime \prime} e \sin (E)\right) .
\end{gathered}
$$

To solve the problem it is necessary to impose that (1) and (4) have the same values, because $M_{2}$ and $M_{3}$ are in the same initial position. Remember that the angular velocity $(\omega)$ of the system is unit; so, $\psi$ can be considered to be the time as well as the angle. So, $t=\psi$ and $E=\eta$ (eccentric anomaly), where

$$
\begin{gathered}
\cos (\psi)=\varepsilon a\left(\varepsilon^{\prime} \cos (\eta)-e\right), \\
\sin (\psi)=\varepsilon \varepsilon^{\prime} a \sqrt{1-e^{2}} \varepsilon^{\prime \prime} \sin (\eta), \\
\psi=a^{3 / 2}\left(\eta-\varepsilon^{\prime \prime} e \sin (\eta)\right) .
\end{gathered}
$$

This set of equations will be used to generate the solutions of the problem. Hénon's problem [35], formulated as Lambert's problem, can be described in the way shown next $[36,37]$.

(i) The position of $M_{3}$ is known at $t=\psi_{0}$ (point $P$, initial point of the transfer orbit). The position $R_{1}$ can be specified as a function of the angle $\psi_{0}$ by

$$
R_{1}=\frac{a\left(1-e^{2}\right)}{1+e \cos \left(\psi_{0}\right)} .
$$

It is the same value for $M_{2}$ and $M_{3}$, because $M_{2}$ and $M_{3}$ occupy the same position at the initial moment $\left(t=\psi_{0}\right)$.

(ii) The position of $M_{3}$ is also known at $t=\psi_{f}$ (point $Q$, the final point of the transfer orbit). The position $R_{2}$ is described by the equation

$$
R_{2}=\frac{a\left(1-e^{2}\right)}{1+e \cos \left(\psi_{f}\right)} .
$$

(iii) The total transfer time is given by $\Delta t=\omega\left(\psi_{f}-\psi_{0}\right)$.

(iv) The total angle $\Delta \psi$, between the points $P$ and $Q$, for the case where the orbits are elliptic, has several possible values.

First of all, it is necessary to consider two possible choices for the transfer; the first one uses the sense of the shortest possible angle between $P$ and $Q$ ("short way"), and the second one uses the sense of the longest possible angle between these two points ("long way").

After considering these two choices, it is also necessary to consider the possibilities of multirevolution transfers. In this case, the spacecraft leaves the point $P$, makes one or more complete revolutions around $M_{1}$, and then it goes to the point $Q$. Thus, the possibilities, after combining those two factors, indicate that the values for $\Delta \psi$ are $\left[\psi_{f}-\psi_{0}+2 m \pi\right]$ and $\left[2 \pi-\left(\psi_{f}-\psi_{0}\right)+2 m \pi\right]$. There is no upper limit for $m$, and this problem has an infinite number of solutions, except in the case where the orbit of $M_{3}$ is parabolic or hyperbolic, where $\Delta \psi$ has a unique value.

Lambert's problem solution is the Keplerian orbit that contains the points $P$ and $Q$ and that requires the given transfer time $\Delta t=\Delta \psi=\psi_{f}-\psi_{0}$ for the spacecraft to travel between those two points. In this paper, Gooding's Lambert routine is used to solve Lambert's problem [62].

Possible applications for this technique are interplanetary research in the Solar System, a basis for a transportation system between the Earth $\left(M_{1}\right)$ and the Moon $\left(M_{2}\right)$ where no orbit correction is required, and so forth.

To get the velocity variation $(\Delta V)$, the following steps are used.

(1) Find the radial and the transverse components of the velocity vector of $M_{2}$ at the points $P$ and $Q$. They are also the velocity components of $M_{3}$ immediately before the first impulse and immediately after the second impulse, respectively, due to the fact that their orbits intercept on those points. The following steps are used to get the [43]

(a) radial velocity

$$
V_{r}=\frac{e \sin (\nu)}{\sqrt{a\left(1-e^{2}\right)}}
$$

(b) transverse velocity

$$
V_{t}=\frac{1+e \cos (\nu)}{\sqrt{a\left(1-e^{2}\right)}}
$$

where $v$ is the true anomaly.

(2) Solve Lambert's Problem for a transfer between the points $P$ and $Q$. At this instant the transfer time is also known.

(3) After obtaining the components of the velocity vector immediately before and after the impulses, it is possible to calculate the magnitude of the two impulses $\left(\Delta V_{1}\right.$ and $\left.\Delta V_{2}\right)$ and to add them to obtain the total impulse required for transfer $(\Delta V)$.

\section{Numerical Solutions for the Multiple Encounters Problem}

To make numerical simulations, the procedure is to vary the initial and final positions of $M_{2}$ for the maneuver. Then, the values of the velocity variation and other quantities can be calculated to find the maneuvers with the minimum consumption. The make the results easier to visualize, it is interesting to show the results considering a fixed value of 
$\psi_{0}$. Then, the solutions are given in terms of $\psi_{f}\left(\psi_{f} \in\right.$ $\left.\left[\psi_{0}, \psi_{s}\right]\right)$, which is the angle that defines the final point of the maneuver. $\psi_{s}$ is the maximum value for $\psi_{f}$. The range of these parameters is provided as an input of the simulation. For each set of the simulated maneuvers, the figures show the solutions of the Multiple Encounters Problem.

3.1. Simulation for the Parameters $\psi_{o}=-2.5$ and $\psi_{o} \leq$ $\psi_{f} \leq \psi_{s}=3.5 \mathrm{rad}$. The procedure for the simulation is the following.

(1) Values of the eccentricities of the primaries, $M_{1}$ and $M_{2}$, are defined. It means that the dynamical system is defined, because the maneuvers are assumed to be planar and the semimajor axis is always unitary, by the definition of the canonical units.

(2) An initial value for the point where the spacecraft leaves the body $M_{2}$ is selected. In the example shown here, this value is $\psi_{0}=-2.5 \mathrm{rad}$. Simulations with different values were made, and the results were very similar.

(3) The angle that determines the point of the return of the spacecraft to $M_{2}, \psi_{f}$, is then varied to cover the desired range for this variable. This condition also defines the duration of the transfer, because the angular velocity is unity; so, $\psi_{f}-\psi_{0}$ also represents the time of the transfer.

(4) Using the time of the transfer and the initial and final points defined earlier, it is possible to solve the associated Lambert's problem. This solution gives the transfer orbit and the total increment of velocity to perform the transfer.

(5) Considering the possibility of several revolutions for the transfer, a family of solutions appears for each pair of points. They are plotted in figures that show the complete set of solutions.

Let us assume the following parameters: $\psi_{o}=-2.5 ; \psi_{o} \leq$ $\psi_{f} \leq 3.5 \mathrm{rad}$. Figure 2 shows the solutions in terms of the true anomaly $(v)$ for the situation where the eccentricity of the primary $\left(M_{2}\right)$ is 0.5 . The horizontal axis shows the value of $\psi_{f}$ in rad, and the vertical axis shows the true anomaly of the final point of the maneuver. Every dot in this plot represents one solution of the problem and so one transfer orbit. Note that the solutions are not unique, because the spacecraft may give several revolutions around the primary. Up to 6 revolutions were included in these simulations.

The same solutions can be seen in Figure 3 but now in terms of the eccentric anomaly $(\eta)$ for the same situation where the eccentricities of the primaries are 0.5. The horizontal axis again shows the value of $\psi_{f}$ in rad, and the vertical axis now shows the eccentric anomaly of the final point of the maneuver. Every dot in this plot also represents one transfer orbit and so one solution of the problem.

Figure 4 shows the total velocity variations for the same solutions, where the eccentricities of the primaries are 0.5. The horizontal axis shows again the value of $\psi_{f}$ in rad, and the vertical axis now shows the total impulse that needs to be

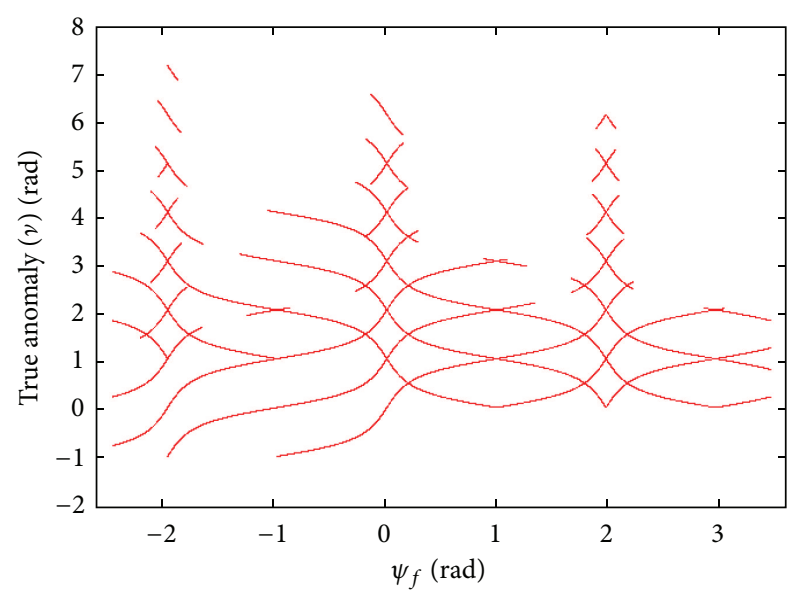

FIGURE 2: Solution in terms of the true anomaly $(\nu) ; e=0.5,-2.5 \leq$ $\psi_{f} \leq 3.5 \mathrm{rad}$.

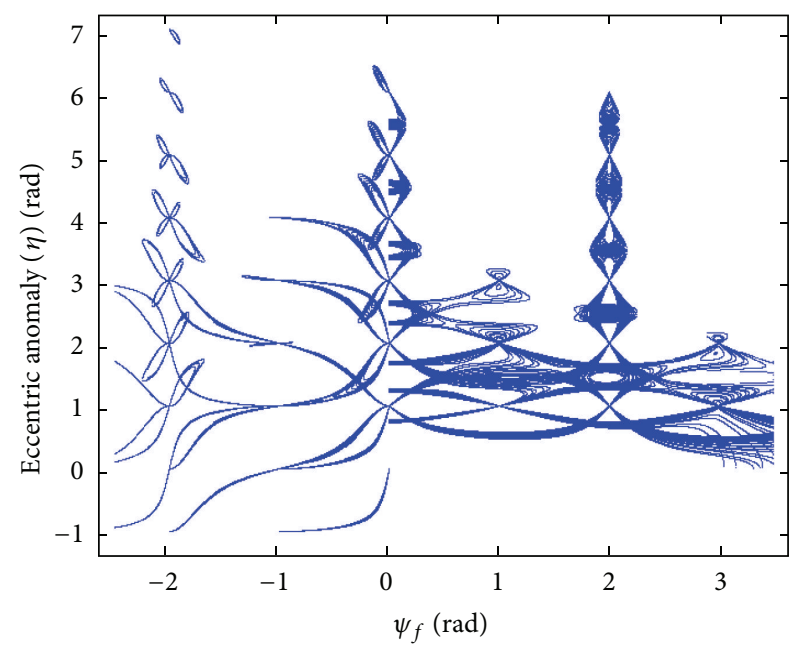

FIGURE 3: Solution in terms of the eccentric anomaly $(\eta) ; e=0.5$, $-2.5 \leq \psi_{f} \leq 3.5 \mathrm{rad}$.

applied to the spacecraft to complete the maneuver. Every dot in this plot also represents one transfer orbit. It is interesting to note the large range of values for the total velocity variation $(\Delta V)$, which goes from values closer to zero to values as high as 7 canonical units. It is important to note that there are values of $\Delta V$ very near to zero; so, the transfer from one body back to the same body near those conditions has a very small impulse to be performed.

Figure 5 shows the semi-major axis for the same solutions. The horizontal axis shows again the value of $\psi_{f}$ in rad, and the vertical axis now shows the semi-major axis of the transfer orbit. Every dot in this plot also represents one transfer orbit.

Several transfer times $\left(\Delta t=\psi_{f}-\psi_{o}\right)$ were simulated, since the initial angle was kept constant. Among numerous transfer orbits, it is shown that there are families of solutions where the $\Delta V$ required for the transfer is very small, close 


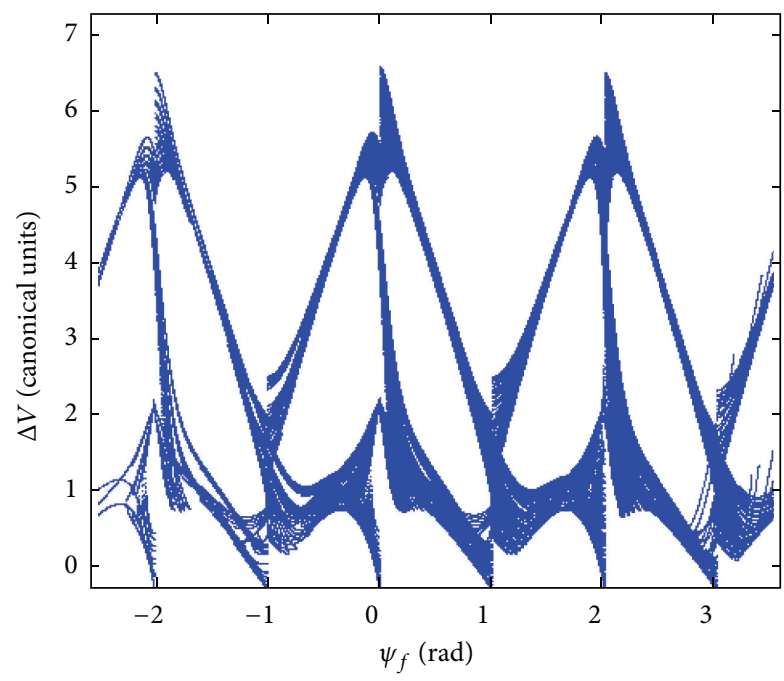

FIGURE 4: Velocity variation $(\Delta V)$ versus $\psi_{f}$, for $e=0.5, \psi_{o}=$ $-2.5 \mathrm{rad}$, and $-2.5 \leq \psi_{f} \leq 3.5 \mathrm{rad}$.

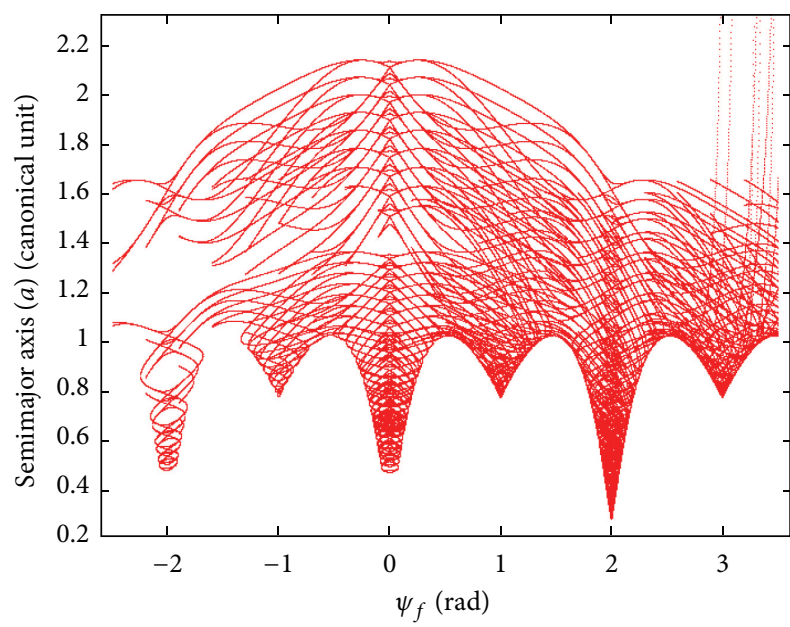

FIGURE 5: Semimajor axis ( $a$ ) versus $\psi_{f}$, for $e=0.5, \psi_{o}=-2.5 \mathrm{rad}$, and $-2.5 \leq \psi_{f} \leq 3.5 \mathrm{rad}$.

to zero. These orbits are good solutions, because the fuel consumption for this transfer is minimal. Solutions with minimum $\Delta V$ (close to zero) provide trajectories that are good candidates for future missions.

Similar simulations were performed for different values of the eccentricity. The results are similar, and so they are omitted here.

The results of those simulations with various eccentricities showed that a system of primaries with smaller eccentricities are better for the execution of Multiple Encounters Orbits, with respect to the fuel consumption, because they are smaller when compared to system of primaries with high eccentricities $(e \rightarrow 1)$. Another point is that the more eccentric the orbit of $M_{3}$, the higher the fuel consumption for the spacecraft to exit and return to the body $M_{2}$. This implies that it is more common to find better solutions (minimal $\Delta V$ )
TABLE 1: Solutions with $\Delta V<0.1$.

\begin{tabular}{|c|c|c|c|c|}
\hline Eccentricity & $\psi(\mathrm{rad})$ & $\begin{array}{c}\text { True } \\
\text { anomaly } \\
\nu \text { (rad) }\end{array}$ & $\begin{array}{c}\text { Eccentric } \\
\text { anomaly } \\
\eta(\mathrm{rad})\end{array}$ & $\begin{array}{c}\text { Velocity } \\
\text { variation } \\
\Delta V\end{array}$ \\
\hline \multirow{4}{*}{$e=0$} & -0.001 & 1.9990 & 1.9990 & 0.000111 \\
\hline & -2.001 & 0.9990 & 0.9990 & 0.000167 \\
\hline & 0.933 & 1.9330 & 1.9330 & 0.000179 \\
\hline & -0.099 & 0.9010 & 0.9010 & 0.000195 \\
\hline \multirow{4}{*}{$e=0.1$} & -0.001 & 1.9988 & 1.9986 & 0.000176 \\
\hline & -2.001 & 0.9988 & 0.9986 & 0.000209 \\
\hline & -0.002 & 1.9975 & 1.9973 & 0.000353 \\
\hline & -2.002 & 0.9975 & 0.9973 & 0.000419 \\
\hline \multirow{4}{*}{$e=0.5$} & -2.001 & 0.9965 & 0.9940 & 0.008940 \\
\hline & -0.001 & 1.9965 & 1.9940 & 0.008940 \\
\hline & 1.999 & 1.9965 & 1.9980 & 0.008946 \\
\hline & 1.999 & 0.9965 & 0.9980 & 0.008964 \\
\hline \multirow{4}{*}{$e=0.9$} & 0.000 & 2.0000 & 2.0000 & 0.007984 \\
\hline & 2.000 & 1.0000 & 1.0000 & 0.007984 \\
\hline & -2.000 & 1.0000 & 1.0000 & 0.012248 \\
\hline & 2.000 & 2.0000 & 2.0000 & 0.012248 \\
\hline
\end{tabular}

among circular orbits $(e=0)$ or among orbits with small eccentricities.

Table 1 shows solutions where the velocity variation $(\Delta V)$ for the Multiple Encounters Problem is minimal considering eccentricities from zero to 0.9 . The existence of solutions is clear with small variation of velocity in all situations, but the minimum values occur for lower values of the eccentricity of the primaries.

\section{The Swing-By Maneuvers}

The dynamics of the two bodies is used in the present formulation. The system is considered to be formed by three bodies. It is possible to say that

(1) the body $M_{1}$, with finite mass, is located in the center of mass of the Cartesian system;

(2) $M_{2}$, a smaller body, can be a planet or a satellite of $M_{1}$, in a Keplerian orbit around $M_{1}$;

(3) a body $M_{3}$, a spacecraft with infinitesimal mass, is traveling in a Keplerian orbit around $M_{1}$, when it makes an encounter with $M_{2}$.

This encounter changes the orbit of $M_{3}$ with respect to $M_{1}$ and, by the hypothesis assumed for the problem, it is considered that the orbits of $M_{1}$ and $M_{2}$ do not change.

Using the "patched conics" approximation, the equations that quantify those changes are available in the literature [50]. The standard maneuver can be identified by the following three parameters (Figure 6):

(i) $\left|\vec{V}_{\infty}\right|$, the magnitude of the velocity of the spacecraft with respect to $M_{2}$ when approaching the celestial body; 


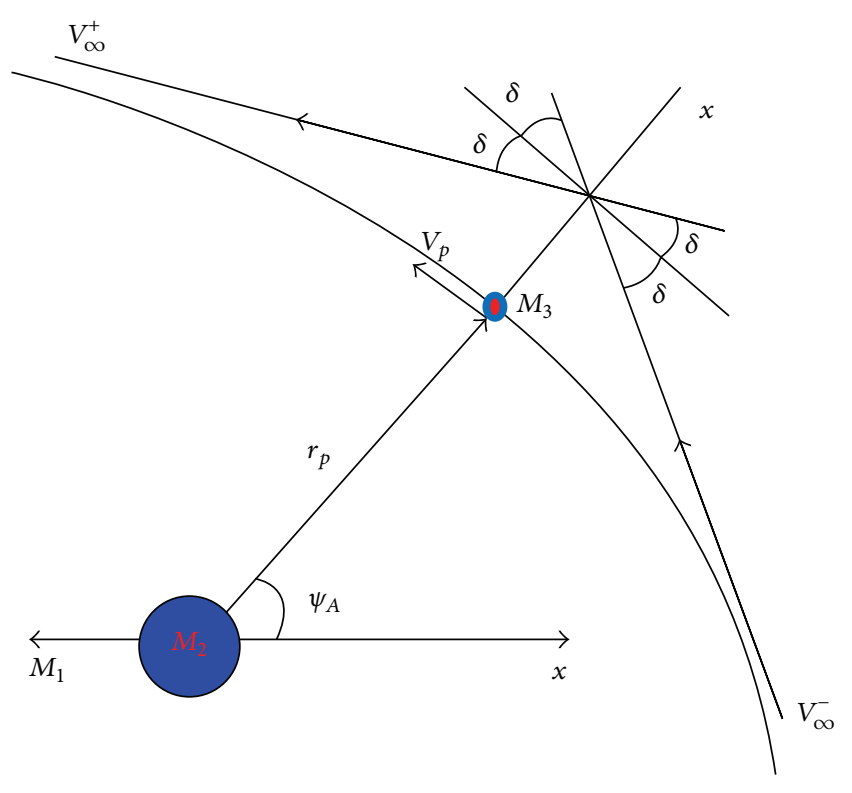

Figure 6: Swing-by maneuver.

(ii) $r_{p}$, the distance between the spacecraft and the celestial body during the closest approach;

(iii) $V_{p}$, the absolute value of the velocity at the periapsis;

(iv) $\psi_{A}$, the angle the approach, that is, the angle between the line connecting the primaries and the periapsis of the close approach trajectory.

Having those variables, it is possible to obtain $\delta$, that is, half of the total deflection angle, by using the equation [50]

$$
\delta=\arcsin \left(\frac{1}{1+r_{p} V_{\infty}^{2} / \mu_{2}}\right) .
$$

A complete description of this maneuver and the derivation of the equations can be found in Broucke [50]. The final equations are reproduced as follows:

$$
\begin{gathered}
\Delta E=-2 V_{2} V_{\infty} \sin (\delta) \sin \left(\psi_{A}\right), \\
\Delta C=-2 V_{2} V_{\infty} \sin (\delta) \sin \left(\psi_{A}\right), \\
\Delta V=|\Delta \vec{V}|=2\left|\vec{V}_{\infty}\right| \sin (\delta)=2 V_{\infty} \sin (\delta) .
\end{gathered}
$$

4.1. Hypotheses for a Swing-By with Jupiter (JGA-Jupiter Gravity Assisted) Obtained by Solving the Multiple Encounters Problem. To solve this problem, the following assumptions are made.

(1) The system is formed by two bodies in elliptic orbits around their mutual center of mass and a third massless body moving under the action of the gravitational forces.

(2) The origin of the system is placed in the center of mass. The horizontal axis is the line connecting the bodies $M_{1}$ and $M_{2}$ and the vertical axis is perpendicular to that line.
(3) The spacecraft leaves the point $P$ (see Figure 1), crosses the horizontal line (Sun-Jupiter), goes to the apsis, and then reaches the point $Q$, where the close approach occurs (see now Figure 6).

(4) After the close approach, the spacecraft modifies the velocity, energy, and angular momentum (11) to (13).

(5) We used the canonical system of units. This formulation implies that the unit of distance is the distance between $M_{1}$ (Sun) and $M_{2}$ (Jupiter); the angular velocity $(\omega)$ of the motion of $M_{1}$ and $M_{2}$ is assumed to be unitary; the mass of the smaller primary $\left(M_{2}\right)$ is given by $\mu=m_{2} /\left(m_{1}+m_{2}\right)$ (where $m_{1}$ and $m_{2}$ are the real masses of $M_{1}$ and $M_{2}$, resp.), and the mass of $M_{2}$ is $(1-\mu)$, to make the total mass of the system unitary; the gravitational constant is one.

In this system of units, the gravitational parameter of Jupiter is $\mu_{j}=9.47368421 \times 10^{-2}$. More details are available in [43].

4.2. Swing-By with $e=0.1, \psi_{o}=-2.5$, and $\psi_{o} \leq$ $\psi_{f} \leq 3.5 \mathrm{rad}$. Numerical simulations were performed using Jupiter for the body from where the spacecraft escapes and performs the swing-by in the return trip. This situation can happen in a practical situation where it is desired to use the gravity of Jupiter to help to move a spacecraft that is around Jupiter to another location in the space, like another planet, asteroid, and so forth. The velocity $(\Delta V)$ and the energy $(\Delta E)$ variations, which depend on the final angle of the maneuver $\psi_{f}$, are analyzed after the Jupiter Gravity Assisted (JGA). Among the simulation parameters, the approach distance of the spacecraft during the swing-by is an important parameter. In order to get the strongest possible results without taking the risk of colliding the spacecraft into the atmosphere of Jupiter, a value of 1.2 Jupiter radius $\approx 5.725019385545 \times$ $10^{-4}$ was used. Table 2 shows the solutions with maximum variations of velocity and energy.

The numerical results show that the largest gain for the velocity variation $(\Delta V)$ does not imply the largest gain in energy $(\Delta E)$, which is a consequence of (11) to (13). Note that the variation in energy has an extra term $\left(\sin \left(\psi_{A}\right)\right)$, affecting the magnitude of the change in energy and not the magnitude of the change in velocity. This angle is determined by the geometry of the approach, which is defined by the choices of the initial and final points of the Multiple Encounters Maneuver. Figure 7 shows the variation of velocity $(\Delta V)$ obtained from the swing-by for the situation where the eccentricity of the primaries is 0.1 , the initial position of the maneuver is specified by the angle $\psi_{o}=-2.5 \mathrm{rad}$, and the final position of the maneuver (angle $\psi_{f}$ ) is in the range between -2.5 and $3.5 \mathrm{rad}$. Note that there are positions with very sharp maximums. Figure 8 shows the equivalent variation of the energy $(\Delta E)$. Note that, in general, the maximums are in the same position, but there are slight differences between both plots, for the reasons already explained. Varying the values of the angles $\left(\psi_{0}\right)$, values of maximum gains for the variation of the energy $(\Delta E)$ and velocity $(\Delta V)$ were found. The variation 
TABLE 2: Swing-by with $\Delta V>1.2, \Delta V$, and $\Delta E$ in canonical units.

\begin{tabular}{|c|c|c|c|c|c|}
\hline \multicolumn{2}{|c|}{$\begin{array}{c}e=0.1 \\
\psi_{o}=-2.5 \mathrm{rad} \\
\psi_{s}=3.5 \mathrm{rad}\end{array}$} & \multicolumn{2}{|c|}{ Angle of approach } & Variation of velocity & \multirow[t]{2}{*}{ Variation of energy } \\
\hline $\mathrm{rad}$ & Degree & $\mathrm{rad}$ & Degree & $\Delta V$ & \\
\hline 2.999 & 171.830043 & 3.8745 & 221.992498 & 1.2159 & 0.81346 \\
\hline 3.001 & 171.944634 & 3.8770 & 222.135737 & 1.2144 & 0.81472 \\
\hline-1.001 & -57.3530753 & 3.8902 & 222.892041 & 1.2053 & 0.82042 \\
\hline 1.999 & 114.534263 & 3.8795 & 222.278977 & 1.2119 & 0.81532 \\
\hline 2.001 & 114.648855 & 3.8801 & 222.313354 & 1.2116 & 0.81562 \\
\hline 2.999 & 171.830043 & 3.8607 & 221.201816 & 1.2222 & 0.80505 \\
\hline 3.001 & 171.944634 & 3.8607 & 221.201816 & 1.2222 & 0.80509 \\
\hline 1.999 & 114.534263 & 3.8882 & 222.77745 & 1.2113 & 0.82266 \\
\hline 2.001 & 114.648855 & 3.8871 & 222.714425 & 1.2119 & 0.82209 \\
\hline 2.999 & 171.830043 & 3.8857 & 222.63421 & 1.2125 & 0.82121 \\
\hline 1.999 & 114.534263 & 3.8900 & 222.880582 & 1.2067 & 0.82113 \\
\hline 2.999 & 171.830043 & 3.8857 & 222.63421 & 1.2125 & 0.82121 \\
\hline 3.001 & 171.944634 & 3.8817 & 222.405027 & 1.2146 & 0.81913 \\
\hline 1.999 & 114.534263 & 3.8900 & 222.880582 & 1.2067 & 0.82113 \\
\hline 1.999 & 114.534263 & 3.8882 & 222.77745 & 1.2113 & 0.82266 \\
\hline 2.001 & 114.648855 & 3.8871 & 222.714425 & 1.2119 & 0.82209 \\
\hline 2.999 & 171.830043 & 3.8607 & 221.201816 & 1.2222 & 0.80505 \\
\hline 3.001 & 171.944634 & 3.8607 & 221.201816 & 1.2222 & 0.80509 \\
\hline
\end{tabular}

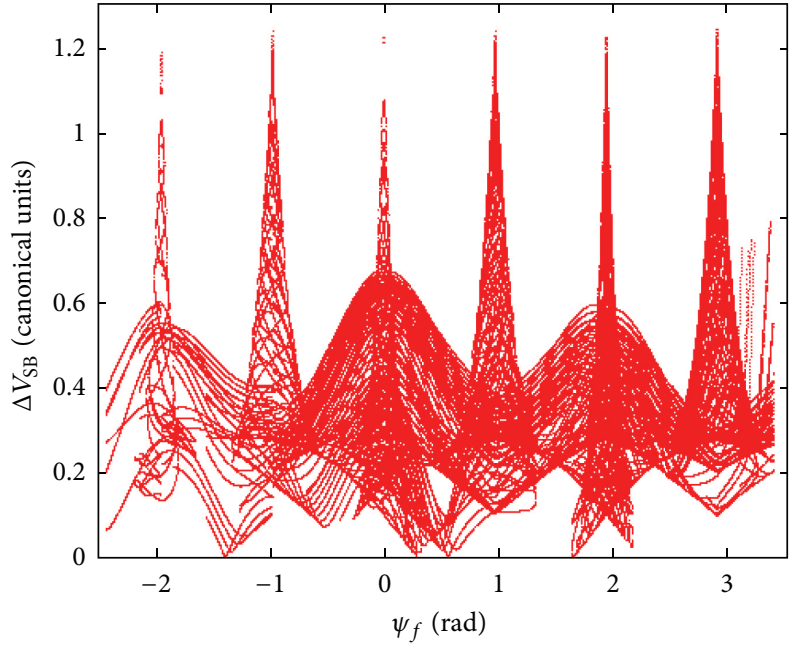

Figure 7: Variation of velocity $(\Delta V)$ obtained by the swing-by for the situation where $e=0.1, \psi_{o}=-2.5 \mathrm{rad}$, and $-2.5 \leq \psi_{f} \leq 3.5 \mathrm{rad}$.

of the velocity $(\Delta V)$ can also be visualized in Table 2 . It is visible that there are several points of maximum gains.

Figures 9 and 10 show the balance between the velocity variations. The total $\Delta V$ is the difference between the value gained from the swing-by and the value spent to maneuver the spacecraft. Figure 9 shows that there are some negative solutions. It means, physically, that the variation of velocity to

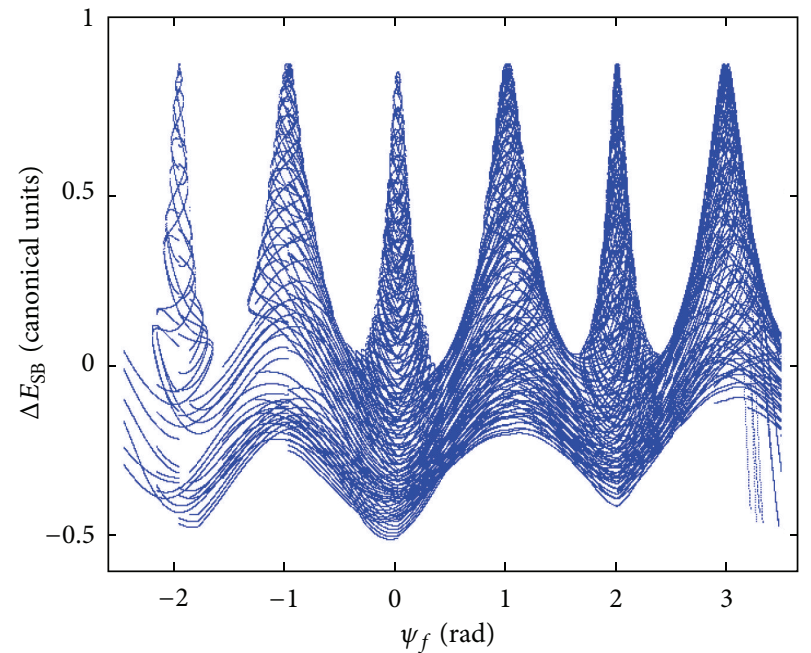

FIGURE 8: Variation of velocity $(\Delta E)$ obtained by the swing-by in the situation where $e=0.1, \psi_{o}=-2.5 \mathrm{rad}$, and $-2.5 \leq \psi_{f} \leq 3.5 \mathrm{rad}$.

maneuver the spacecraft is larger than the gain obtained from the swing-by and the maneuver should not be performed. Positive values mean that the maneuver should be done. Of course, it is interesting to look for the situations with maximum value for this maneuver. Figure 10 is an amplification of Figure 9, considering only the positive results. 


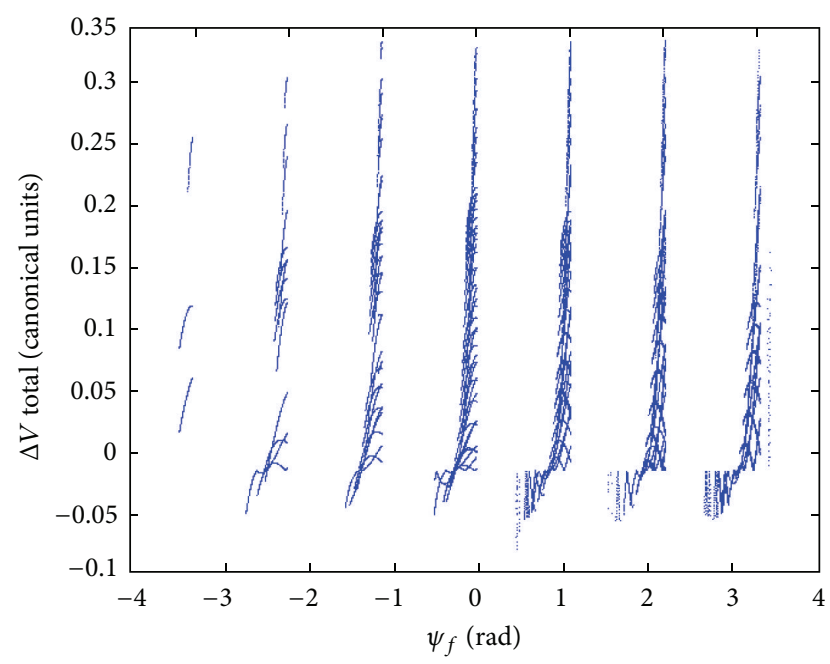

FIGURE 9: Total $\Delta V\left(\Delta V_{\mathrm{SB}}-\Delta V\right)$ obtained by the swing-by for the case where $e=0, \psi_{o}=-\pi \mathrm{rad}$, and $-\pi \leq \psi_{f} \leq \pi \mathrm{rad}$.

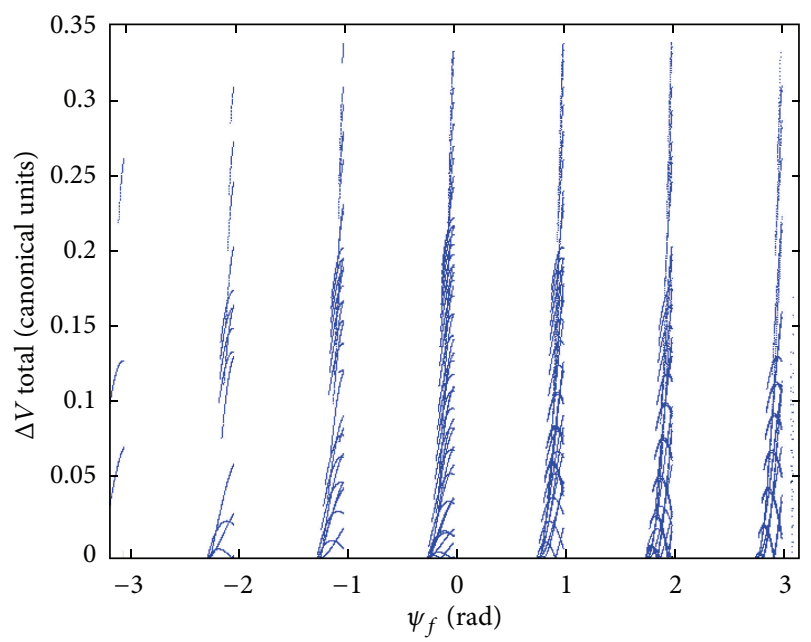

Figure 10: Positive balance in $\Delta V$, obtained from the swing-by in the situation where $e=0, \psi_{o}=-\pi \mathrm{rad}$, and $-\pi \leq \psi_{f} \leq \pi \mathrm{rad}$.

In the simulations performed in this study, some results available in the literature [50], which helps in validating the calculations performed here were also confirmed.

(i) If the swing-by occurs behind the body $M_{2}\left(180^{\circ}<\right.$ $\psi_{A}<360^{\circ}$ ), there will be an energy gain (Table 2 and Figures 10 and 12).

(ii) If the swing-by occurs in front of the body $M_{2}\left(0^{\circ}<\right.$ $\psi_{A}<180^{\circ}$ ), there will be an energy loss (Figures 10 and 12).

Next, Figures 11 and 12 show the importance of the angle of approach $\left(\psi_{A}\right)$ in the swing-by maneuver. For a specific value of the eccentricity of the primaries and a fixed pair of points to start and finish the maneuver $(e=0.1$, $\psi_{o}=-2.5 \mathrm{rad}$, and $\psi_{f}=3.5 \mathrm{rad}$ ), the variations of velocity (Figure 11) and energy (Figure 12) are shown as a function of

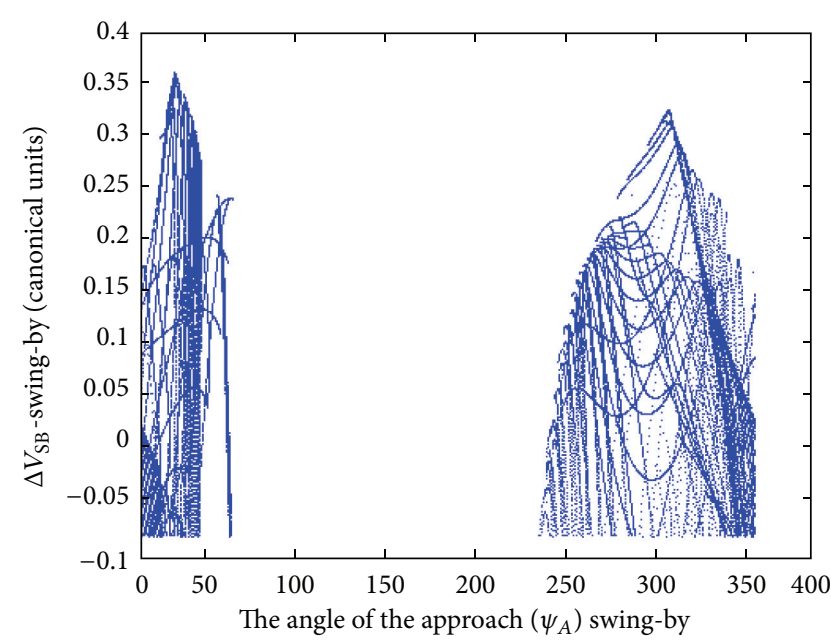

FIgURE 11: $\Delta V$ obtained from the swing-by for the situation where $e=0.1, \psi_{o}=-2.5 \mathrm{rad}$, and $\psi_{f}=3.5 \mathrm{rad}$.

the angle of approach $\left(\psi_{A}\right)$. The scales of the vertical axis are chosen to show the more interesting regions of the plots.

An important point to remember is to look at the type of the orbit of the spacecraft before and after the swing-by (circular, elliptic, hyperbolic, and parabolic). From the two body-problem, it is known that

(i) when the energy of the spacecraft is negative, the orbit is closed (circular or elliptical) and the spacecraft always remains at a finite distance from the central body, which means that the body remains orbiting $\mathrm{M}_{2}$;

(ii) when the energy of the spacecraft is positive, the orbit is open (hyperbolic) and the distance to the central body tends to infinity with the time, and so the body does not remain orbiting $M_{2}$.

It is also important to remember that is the sum of the initial spacecraft energy plus the variation obtained from the swing-by $\left(E_{i}+\Delta E\right)$ that will establish its orbit after the maneuver, where $E_{i}$ is the energy of the spacecraft before the swing-by. So, positive and negative variations are not the only quantity to look for to see how the orbit of the spacecraft will be after the swing-by.

Next, another important question is studied related to this problem, which are the effects of the eccentricity of the primaries in the result of the swing-by maneuver in the return passage by $M_{2}$. It is necessary to remember that the variation of energy obtained from the swing-by has a strong dependence on the geometry of the close approach, a fact that is expressed by the angle of approach in the analytical equations (11) to (13). The eccentricity of the primaries has a direct impact in this geometry, with lower values for the eccentricity generating trajectories for $M_{3}$ that reaches $M_{2}$ in approaches that are near parallel to the trajectory of $M_{2}$. This fact generates values near zero or $\pi$ for $\psi_{A}$, which generates smaller values for the variation of energy. In the opposite side, values near 1.0 for the eccentricity of the primaries generate trajectories for $M_{3}$ that reaches $M_{2}$ in approaches that are 
TABLE 3: Swing-by with $\Delta E>1.148$.

\begin{tabular}{|c|c|c|c|c|c|}
\hline \multicolumn{2}{|c|}{$\begin{array}{c}e=0.9 \\
\psi_{o}=-2.5 \mathrm{rad} \\
\psi_{f}=3.5 \mathrm{rad}\end{array}$} & \multicolumn{2}{|c|}{ Angle of approach } & Variation of velocity & Variation of energy \\
\hline $\mathrm{rad}$ & Degree & $\mathrm{rad}$ & Degree & $\Delta V$ & $\Delta E$ \\
\hline 2.292 & 131.32193 & 4.82564 & 276.48892 & 1.15561 & 1.14820 \\
\hline 2.293 & 131.37922 & 4.82347 & 276.36436 & 1.15533 & 1.14821 \\
\hline 2.294 & 131.43652 & 4.82130 & 276.23991 & 1.15505 & 1.14821 \\
\hline 2.295 & 131.49381 & 4.81913 & 276.11552 & 1.15478 & 1.14821 \\
\hline 2.296 & 131.55111 & 4.81696 & 275.99125 & 1.15451 & 1.14821 \\
\hline 2.297 & 131.60841 & 4.81479 & 275.86709 & 1.15425 & 1.14820 \\
\hline
\end{tabular}

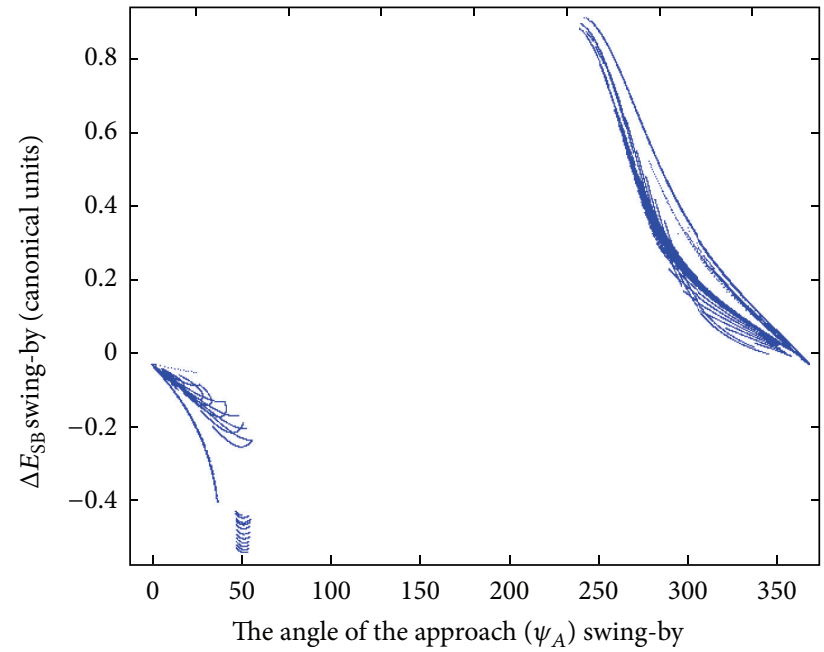

FIgURE 12: $\Delta E$ obtained by the swing-by for the situation where $e=$ $0.1, \psi_{o}=-2.5 \mathrm{rad}$, and $\psi_{f}=3.5 \mathrm{rad}$.

near perpendicular to the trajectory of $M_{2}$. This fact generates values near $\pi / 2$ or $3 \pi / 2$ for $\psi_{A}$, which generates larger values for the variation of energy. To verify if this expected situation occurs, a study is made assuming 0.9 for the eccentricity of the primaries. Figures 13 and 14 show the results. For a value of the eccentricity of the primaries of 0.9 and a fixed point to start the maneuver $\left(\psi_{o}=-2.5 \mathrm{rad}\right)$, the variation of energy (Figure 11) is shown as a function of the point to finish the maneuver $\left(\psi_{f}\right)$. It is noticed that the maximum values reached is larger than in the situation where $e=0.1$ (Figure 8).

Figure 14 is basically the variation of energy also as a function of the point to start the maneuver $\left(\psi_{o}\right)$, in a threedimensional graph. It is noted that the figure is basically composed of similar planar figures that are arranged after each other. This is the main reason why the present paper concentrates in showing results for a fixed value of the point to start the maneuver $\left(\psi_{o}\right)$. It is much easier to visualize the results, and there is no loss of information. Table 3 shows the detailed results for the points with maximum variation of energy. It is interesting to note that the increase of the eccentricity of the primaries increases the cost of the initial

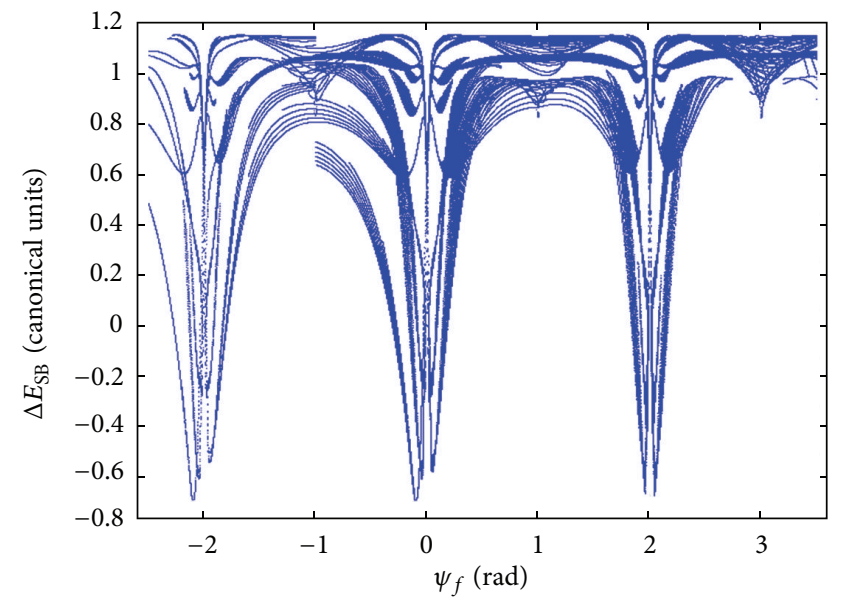

Figure 13: Variation of energy $(\Delta E)$ obtained by the swing-byin the situation where $e=0.9, \psi_{o}=-2.5 \mathrm{rad}$, and $-2.5 \leq \psi_{f} \leq 3.5 \mathrm{rad}$.

maneuver of the spacecraft, but it also increases the gains from the swing-by. The next result shows that the gains are larger and the increase of the eccentricity is benefic for the whole maneuver.

\section{Conclusion}

Optimum space maneuvers were studied, which the goal of finding trajectories that reduces the fuel consumption for interplanetary missions. In particular, in situations where the spacecraft leaves and comes back to the same body (Multiple Encounters Problem), considering nonsymmetrical situations for the start and finish of the maneuver. Several simulations were performed and shown, and the solutions found low-cost maneuvers that can be used for the planning of real missions.

Then, the study included swing-by maneuvers in the return passage by the mother body as a form of gaining energy. The numerical simulations showed that the velocity $(\Delta V)$ and the energy $(\Delta E)$ variations increase with the eccentricity of the orbit of the primaries. This fact is interesting, because it contrasts with the fact that the increase of this eccentricity generates initial maneuvers with higher costs. 


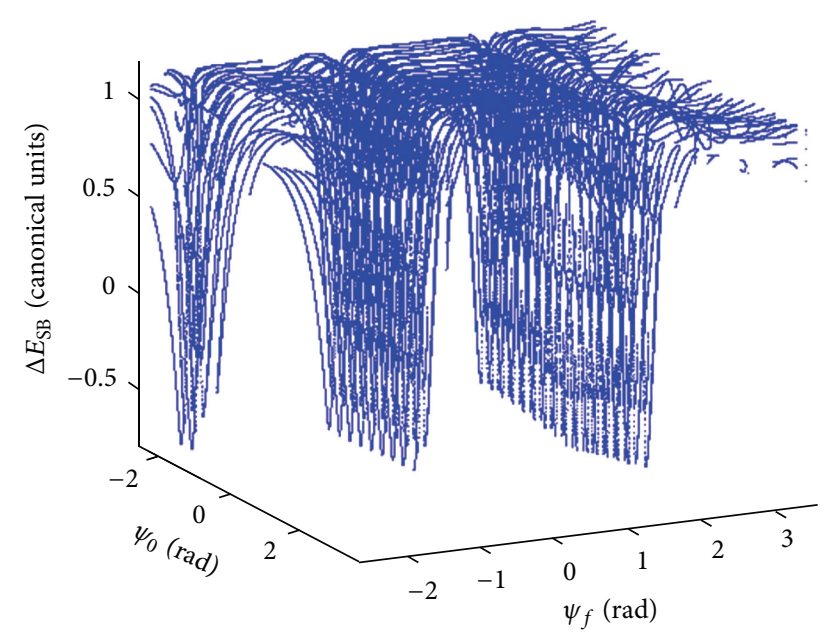

FIgURE 14: Variation of energy $(\Delta E)$ obtained by the swing-by in the situation where $e=0.9,-2.5 \leq \psi_{o} \leq 3.5 \mathrm{rad}$, and $-2.5 \leq \psi_{f} \leq$ $3.5 \mathrm{rad}$.

The simulations showed regions where the net result is positive.

It was also shown that the gravity assisted maneuvers made with the Jupiter Planet (JGA) can provide a considerable variation of velocity and energy for a spacecraft, thus reducing the costs of the mission. In the figures and tables shown in this work, it is verified that this maneuver is a powerful tool that can also be used in interplanetary missions that require that the spacecraft leaves a body and returns later to this same body. This is interesting for a maneuver that needs to move a spacecraft that is around Jupiter to send it to another location of the Solar System or beyond.

\section{Acknowledgments}

This work was accomplished with the support of São Paulo State Science Foundation (FAPESP) under Contracts 2009/16517-7, 2011/09310-7, and 2011/08171-3, CAPES, CNPq (contract 304700/2009-6), and INPE-National Institute for Space Research-Brazil.

\section{References}

[1] R. H. Goddard, A Method of Reaching Extreme Altitudes, vol. 71, Smithsonian Institute Public Miscellanea Collect, 1919.

[2] W. Hohmann, Die Erreichbarkeit der Himmelskorper, Oldenbourg, Munique, Germany, 1925.

[3] A. Shternfeld, Soviet Space Science, Basic Books, New York, NY, USA, 1959.

[4] R. F. Hoelker and R. Silber, "The Bi-Elliptic Transfer Between Circular Co-Planar Orbits," Tech Memo 2-59, Army Ballistic Missile Agency, Redstone Arsenal, Ala, USA, 1959.

[5] D. F. Bender, "Optimum coplanar two-impulse transfers between elliptic orbits," Aerospace Engineering, pp. 44-52, 1962.

[6] K. Eckel, "Optimum transfer in a central force field with $\mathrm{n}$ impulses," Astronautica Acta, vol. 9, no. 5-6, pp. 302-324, 1963.
[7] C. Marchal, "Transferts optimaux entre orbites elliptiques coplanaires (Durée indifférente)," Astronautica Acta, vol. 11, no. 6, pp. 432-445, 1965.

[8] F. W. Gobetz and J. R. Doll, "A survey of impulsive trajectories," AIAA Journal, vol. 7, pp. 801-834, 1969.

[9] J. E. Prussing, "Optimal four-impulse fixed-time rendezvous in the vicinity of a circular orbit," AIAA Journal, vol. 7, no. 5, pp. 928-935, 1969.

[10] J. E. Prussing, "Optimal two- and three-impulse fixed-time rendezvous in the vicinity of a circular orbit," AIAA Journal, vol. 8, no. 7, pp. 1221-1228, 1970.

[11] D. J. Jezewski and D. Mittleman, "An analytic approach to twofixed-impulse transfers between Keplerian orbits," Journal of Guidance, Control, and Dynamics, vol. 5, no. 5, pp. 458-464, 1982.

[12] K. G. Eckel, "Optimal impulsive transfer with time constraint," Acta Astronautica, vol. 9, no. 3, pp. 139-146, 1982.

[13] J. E. Prussing and J. H. Chiu, "Optimal multiple-impulse timefixed rendezvous between circular orbits," Journal of Guidance, Control, and Dynamics, vol. 9, no. 1, pp. 17-22, 1986.

[14] H. Jin and R. G. Melton, "Transfers between circular orbits using fixed impulses. AAS paper 91-161," in Proceedings of the AAS/AIAA Spaceflight Mechanics Meeting, Houston, Tex, USA, 1991.

[15] A. F. B. A. Prado and R. Broucke, "Transfer orbits in the Earthmoon system using a regularized model," Journal of Guidance, Control, and Dynamics, vol. 19, no. 4, pp. 929-933, 1996.

[16] A. F. B. A. Prado, "Traveling between the lagrangian points and the earth," Acta Astronautica, vol. 39, no. 7, pp. 483-486, 1996.

[17] D. P. S. Santos, A. F. B. A. Prado, and I. D. Guido Colasurdo, "Four-impulsive rendezvous maneuvers for spacecrafts in circular orbits using genetic algorithms," Mathematical Problems in Engineering, vol. 2012, Article ID 493507, 16 pages, 2012.

[18] S. S. Fernandes and C. M. P. Marinho, "Optimal two-impulse trajectories with moderate flight time for earth-moon missions," Mathematical Problems in Engineering, vol. 2012, Article ID 971983, 34 pages, 2012.

[19] S. S. Fernandes and C. M. P. Marinho, "Sun influence on two-impulsive Earth-to-Moon transfers," Journal of Aerospace Engineering, Sciences and Applications, vol. 4, pp. 82-91, 2012.

[20] D. F. Lawden, "Minimal rocket trajectories," ARS Journal, vol. 23, no. 6, pp. 360-382, 1953.

[21] D. F. Lawden, "Fundamentals of space navigation," Journal of the British Interplanetary Society, vol. 13, pp. 87-101, 1954.

[22] J. R. Brophy and M. Noca, "Electric propulsion for solar system exploration," Journal of Propulsion and Power, vol. 14, no. 5, pp. 700-707, 1998.

[23] L. Casalino, G. Colasurdo, and D. Pastrone, "Optimal lowthrust escape trajectories using gravity assist," Journal of Guidance, Control, and Dynamics, vol. 22, no. 5, pp. 637-642, 1999.

[24] A. A. Sukhanov and A. F. B. A. Prado, "Constant tangential lowthrust trajectories near on oblate planet," Journal of Guidance, Control, and Dynamics, vol. 24, no. 4, pp. 723-731, 2001.

[25] M. R. Sentinella and L. Casalino, "Genetic algorithm and indirect method coupling for low-thrust trajectory optimization," AIAA No. 064468. 2006.

[26] F. Cacciatore and C. Toglia, "Optimization of orbital trajectories using genetic algorithms," Journal of Aerospace Engineering. Sciences and Applications, vol. 1, no. 1, pp. 58-69, 2008. 
[27] D. P. S. Santos, L. Casalino, G. Colasurdo, and A. F. B. A. Prado, "Optimal trajectories using gravity assisted maneuver and Solar Electric Propulsion (SEP) towards near-earth-objects," in Proceedings of the 4th WSEAS International Conference on Applied and Theoretical Mechanics (MECHANICS '08), pp. 6268, Cairo, Egypt, 2008.

[28] V. M. Gomes, A. F. B. A. Prado, and H. K. Kuga, "Low thrust maneuvers for Artificial Satellites," WSEAS Transactions on Applied and Theoretical Mechanics, vol. 3, no. 10, pp. 859-868, 2008.

[29] J. E. Prussing, "Equation for optimal power-limited spacecraft trajectories," Journal of Guidance, Control, and Dynamics, vol. 16, no. 2, pp. 391-393, 1993.

[30] D. P. S. Santos, L. Casalino, G. Colasurdo, and A. F. B. A. Prado, "Optimal trajectories towards near-earth-objects using solar electric propulsion (sep) and gravity assisted maneuver," Journal of Aerospace Engineering. Sciences and Applications, vol. 1, no. 2, 2009.

[31] S. Da Silva Fernandes and F. D. C. Carvalho, "A first-order analytical theory for optimal low-thrust limited-power transfers between arbitrary elliptical coplanar orbits," Mathematical Problems in Engineering, vol. 2008, Article ID 525930, 30 pages, 2008.

[32] O. C. Winter, D. C. Mourão, C. F. Melo, E. N. Macau, J. L. Ferreira, and J. P. S. Carvalho, "Controlling the eccentricity of polar lunar orbits with low-thrust propulsion," Mathematical Problems in Engineering, vol. 2009, Article ID 159287, 2009.

[33] V. M. Gomes and A. F. B. A. Prado, "Avoiding collisions maneuvers using a stochastic approach," International Journal of Mechanics, vol. 5, no. 3, pp. 148-156, 2011.

[34] D. P. S. Santos, Otimização de trajetórias espaciais com propulsão elétrica solar e manobras gravitacionalmente assistidas [Doutorado em Mecânica Espacial e Controle], Instituto Nacional de Pesquisas Espaciais. São José dos Campos, 2009.

[35] M. Hénon, "Sur les orbites interplanétaires qui rencontrent deux fois la," Bulletin Astronomique, vol. 3, p. 377, 1968.

[36] A. F. B. A. Prado and R. A. Broucke, "The problem of transfer orbits from one body back to the same body," in Advances in the Astronautical Sciences, R. G. Melton, L. J. Wood, R. C. Thompson, and S. J. Kerridge, Eds., vol. 82 of Space Flight Mechanics Part II, pp. 1241-1260, 1993.

[37] A. F. B. A. Prado and R. A. Broucke, "Study of Hénon's orbit transfer problem using the Lambert algorithm," AIAA Journal of Guidance, Control, and Dynamics, vol. 17, no. 5, pp. 1075-1081, 1993.

[38] A. D. Bruno, "On periodic flybys of the moon," Celestial Mechanics, vol. 24, no. 3, pp. 255-268, 1981.

[39] L. M. Perko, "Periodic orbits in the restricted three-body problem: existence and asymptotic approximation," SIAM Journal on Applied Mathematics, vol. 27, no. 1, pp. 200-237, 1974.

[40] D. L. Hitzl and M. Hénon, "Critical generating orbits for second species periodic solutions of the restricted problem," Celestial Mechanics, vol. 15, no. 4, pp. 421-452, 1977.

[41] D. L. Hitzl and M. Hénon, "The stability of second species periodic orbits in the restricted problem $(\mu=0)$," Acta Astronautica, vol. 4, no. 9-10, pp. 1019-1039, 1977.

[42] K. C. Howell, "Consecutive collision orbits in the limiting case $\mu=0$ of the elliptic restricted problem," Celestial Mechanics, vol. 40, no. 3-4, pp. 393-407, 1987.

[43] D. P. S. Santos, Aplicações em manobras espaciais do problema de múltiplos encontros [Mestrado em Mecânica Espacial e Controle],
Instituto Nacional de Pesquisas Espaciais, São José dos Campos, 2009.

[44] D. P. S. Santos, A. F. B. A. Prado, and E. M. Rocco, "The use of consecutive collision orbits to obtain swing-by maneuvers," in Proceedings of the 56th International Astronautical Congress, Fukuoka, Japan, 2005.

[45] G. A. Flandro, "Fast reconnaissance missions to the outer solar system utilizing energy derived from the gravitational field of Jupiter," Astronautica Acta, vol. 12, no. 4, pp. 329-337, 1966.

[46] R. W. Farguhar and D. W. Dunham, "A new trajectory concept for exploring the Earth's geomagnetic tail," Journal of Guidance, Control, and Dynamics, vol. 4, no. 2, pp. 192-196, 1981.

[47] L. A. D’Amario, D. V. Byrnes, and R. H. Stanford, "Interplanetary trajectory optimization with application to Galileo," Journal of Guidance, Control, and Dynamics, vol. 5, no. 5, pp. 465-471, 1982.

[48] R. Farquhar, D. Muhonen, and L.C. Church, "Trajectories and orbital maneuvers for the ISEE-3/ICE comet mission," Journal of Astronautical Sciences, vol. 33, no. 3, pp. 235-254, 1985.

[49] D. Dunham and S. Davis, "Optimization of a multiple LunarSwingby trajectory sequence," Journal of Astronautical Sciences, vol. 33, no. 3, pp. 275-288, 1985.

[50] R. A. Broucke, "The Celestial Mechanics of Gravity Assist," AIAA Paper 88-4220, 1988.

[51] R. L. Dowling, W. J. Kosmann, M. A. Minovitch, and R. W. Ridenoure, "Gravity propulsion research at UCLA and JPL, 1962-1964," in Proceedings of the 41st Congress of the International Astronautical Federation, Dresden, Germany, 1991.

[52] A. F. B. A. Prado, Optimal transfer and swing-by orbits in the two- and three-body problems [Ph.D. thesis], Department of Aerospace Engineering and Engineering Mechanics, University of Texas, Austin, Tex, USA, 1993.

[53] A. F. B. A. Prado and R. A. Broucke, "Transfer orbits in restricted problem," Journal of Guidance Control and Dynamics, vol. 18, no. 3, pp. 593-598, 1995.

[54] A. F. B. A. Prado and R. Broucke, "Effects of atmospheric drag in swing-by trajectory," Acta Astronautica, vol. 36, no. 6, pp. 285290, 1995.

[55] A. F. B. A. Prado, "Powered swingby," Journal of Guidance, Control, and Dynamics, vol. 19, no. 5, pp. 1142-1147, 1996.

[56] A. F. B. A. Prado, "Close-approach trajectories in the elliptic restricted problem," Journal of Guidance, Control, and Dynamics, vol. 20, no. 4, pp. 797-802, 1997.

[57] A. E. Petropoulos, J. M. Longuski, and N. X. Vinh, "Shape-based analytic representations of low-thrust trajectories for gravityassist applications," American Astronautical Society, AAS Paper 99-337, 1999.

[58] A. E. Petropoulos and J. M. Longuski, "Automated design of low-thrust gravity-assist trajectories," AIAA Paper 2000-4033, 2000.

[59] V. M. Gomes and A. F. B. A. Prado, "Swing-By maneuvers for a cloud of particles with planets of the Solar System," WSEAS Transactions on Applied and Theoretical Mechanics, vol. 3, no. 11, pp. 869-878, 2008.

[60] V. M. Gomes and A. F. B. A. Prado, "A study of the impact of the initial energy in a close approach of a cloud of particles," WSEAS Transactions on Mathematics, vol. 9, no. 10, pp. 811-820, 2010.

[61] F. C. Faria Venditti, E. Marconi Rocco, A. F. B. A. Prado, and A. Suhkanov, "Gravity-assisted maneuvers applied in the multi-objective optimization of interplanetary trajectories," Acta Astronautica, vol. 67, no. 9-10, pp. 1255-1271, 2010. 
[62] R. H. Gooding, "A procedure for the solution of Lambert's orbital boundary-value problem," Celestial Mechanics and Dynamical Astronomy, vol. 48, no. 2, pp. 145-165, 1990. 


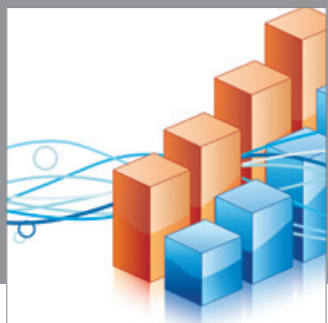

Advances in

Operations Research

mansans

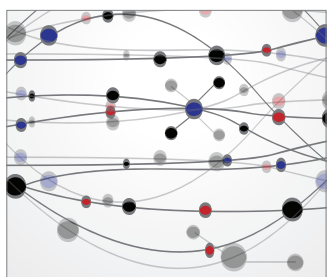

The Scientific World Journal
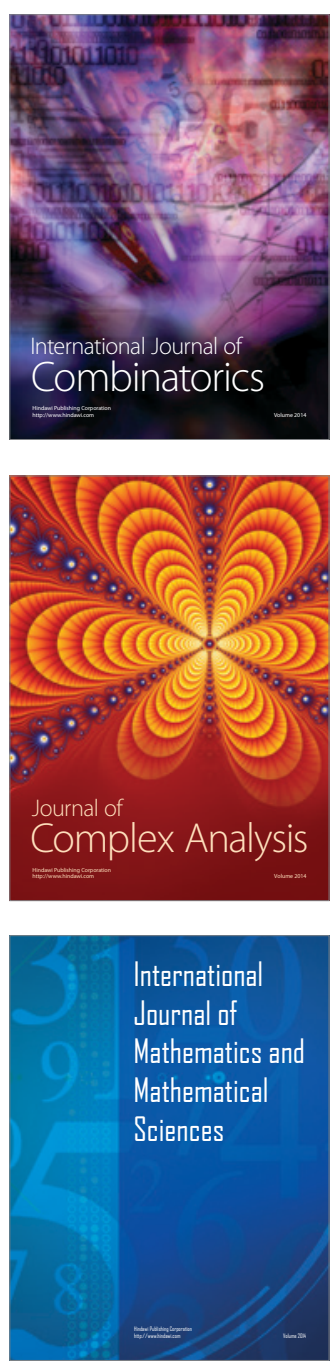
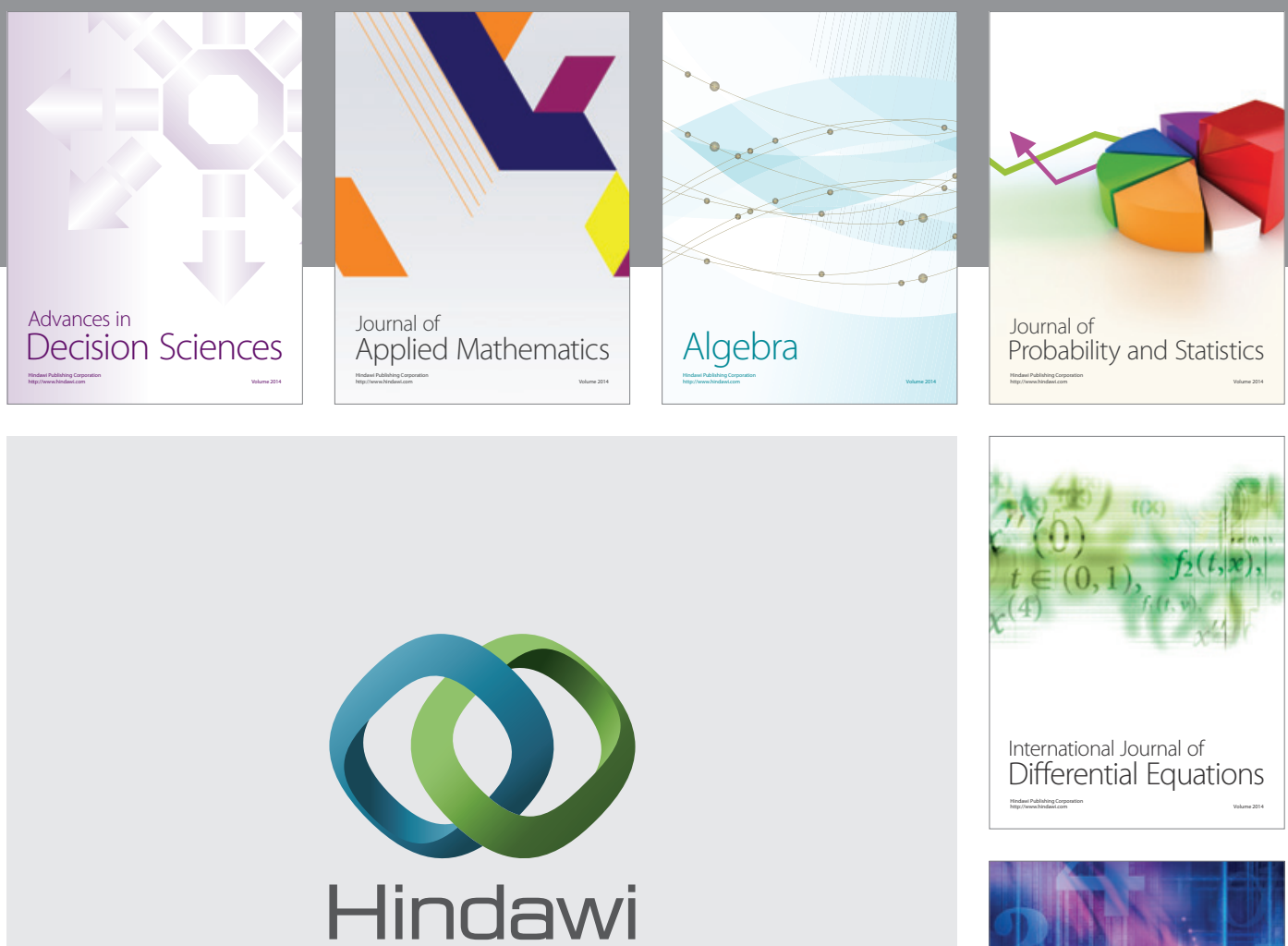

Submit your manuscripts at http://www.hindawi.com
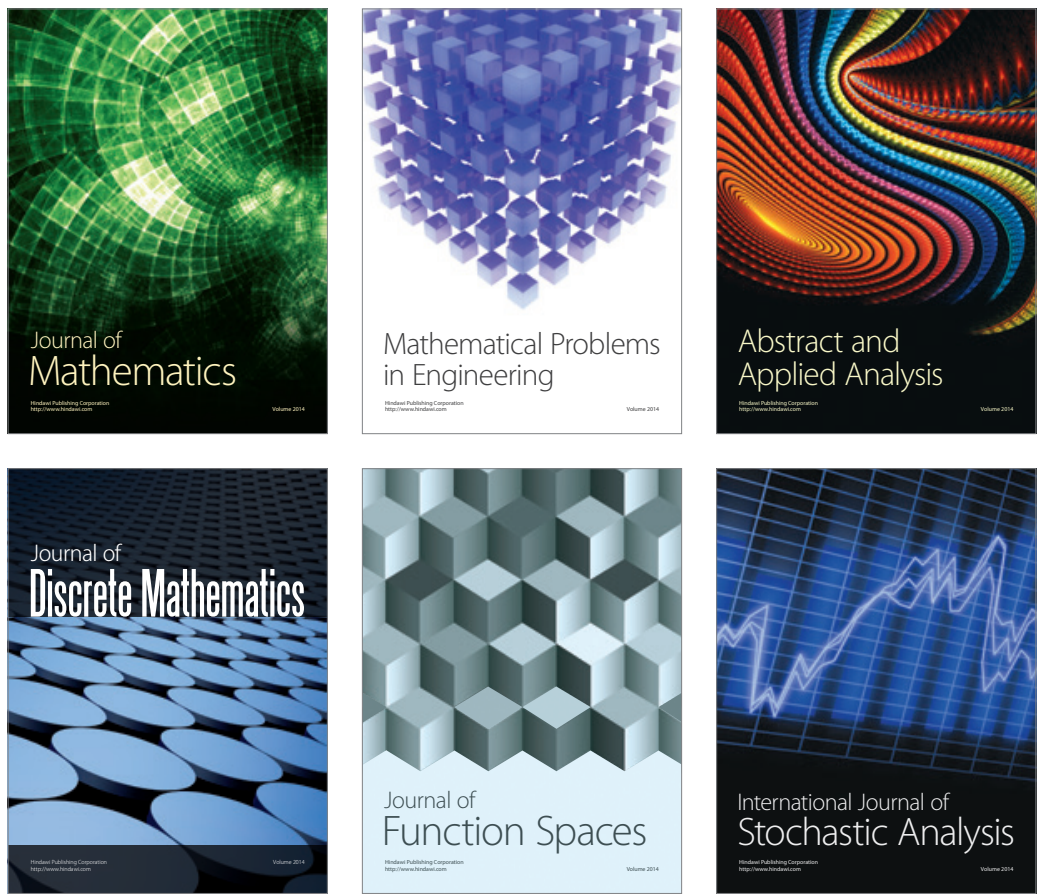

Journal of

Function Spaces

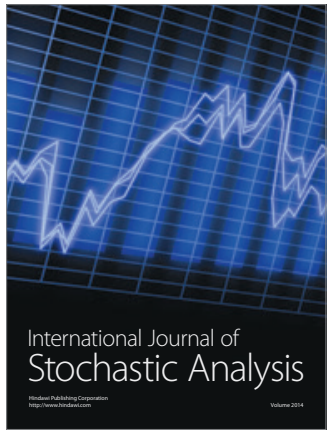

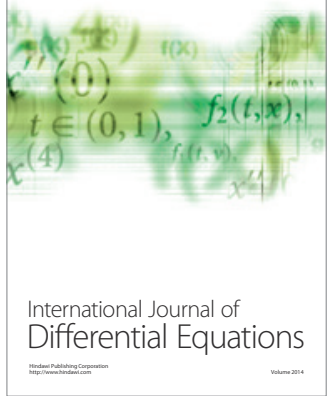
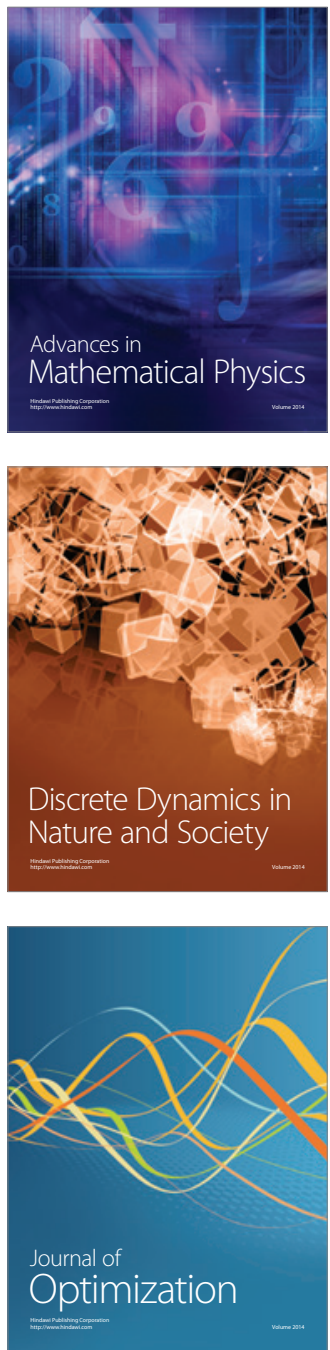